\title{
Nearly full-length genome sequence of a novel astrovirus isolated from chickens with 'white chicks' condition
}

\author{
Joanna Sajewicz-Krukowska ${ }^{1} \cdot$ Katarzyna Domanska-Blicharz $^{1}$ (i)
}

Received: 15 February 2016/Accepted: 16 June 2016/Published online: 23 June 2016

(c) The Author(s) 2016. This article is published with open access at Springerlink.com

\begin{abstract}
Avian astroviruses (aAstVs) are divided into three species, Avastrovirus 1, Avastrovirus 2, and Avastrovirus 3, but there are a few strains are waiting to be assigned to an official taxonomic group. This study presents the molecular characterization of chicken astrovirus (CAstV), PL/G059/2014, which is involved in the induction of "white chicks" condition. The 7382-nucleotidelong genome sequence was determined by next-generation sequencing using an Illumina MiSeq System. Phylogenetic analysis showed that it has the characteristics that are typical of avian astroviruses. However, overall degree of nucleotide sequence identity was $43.6 \%$ to $73.7 \%$ between PL/G059/2014 and other available genome sequences of aAstV strains. The amino acid sequences of the proteins encoded by ORF1a and ORF1b of the studied strain were very similar (86.5-93.8\% identity) to those of CAstVs 4175 and GA2011, but they were only 32.7$35.2 \%$ identical in the case of ORF2, which is used officially for astrovirus species demarcation. These features could suggest that the PL/G059/2014 strain should be assigned to a new species in the genus Avastrovirus. Moreover, the different phylogenetic topology of PL/G059/ 2014 and its nucleotide sequence similarity in different genomic regions could suggest that a recombination event
\end{abstract}

Accession number The nearly full-length genome sequence of PL/ G059/2014 has been submitted to the GenBank database under accession no. KT886453.

Katarzyna Domanska-Blicharz

domanska@piwet.pulawy.pl

1 Department of Poultry Diseases, National Veterinary Research Institute, Al. Partyzantow 57, 24-100 Pulawy, Poland occurred during its evolution and that it has ancestors in common with duck astroviruses.

Keywords Astrovirus - Chicken P Phylogenetic analysis · Recombination

Astroviruses are small viruses with a diameter of approximately $28-30 \mathrm{~nm}$. Their single-stranded RNA genome ranges from 6.8 to $7.9 \mathrm{~kb}$ and contains three open reading frames (ORFs) with a $5^{\prime}$ untranslated region (UTR) and a $3^{\prime}$ UTR, and a poly-A tail. ORF1a encodes the non-structural (NS) polyprotein, while ORF1b and ORF2 encode the RNA-dependent RNA polymerase (RdRp) and the viral capsid structural protein, respectively $[4,7]$. These viruses belong to the family Astroviridae, which is divided into two genera: Mamastrovirus and Avastrovirus, depending on the organisms they infect. For many years, this criterion was also used for further classification of astrovirus strains into separate species. Within the genus Avastrovirus, such division distinguished two turkey astroviruses, turkey astrovirus type 1 (TAstV-1) and type 2 (TAstV-2); two chicken viruses, avian nephritis virus (ANV) and chicken astrovirus (CAstV); and duck astroviruses (DAstV) [15]. However, a number of astroviruses have been found recently in different bird species; moreover, it turned out that they could be transmitted between species [6]. The new official astrovirus classification system is based on the amino acid sequence of viral capsid protein and most of the above-mentioned astroviruses have been assigned to three species: Avastrovirus 1, Avastrovirus 2, and Avastrovirus 3. When this classification was created, due to the lack of available sequences, CAstVs were referred to as "related viruses which may be members of the Avastrovirus genus but have not been approved as species" [8]. In the 
meantime, the full genomes of two CAstV strains and the capsid gene of additional CAstVs from different geographical locations have been sequenced $[3,9,18]$. A phylogenetic analysis of available CAstV ORF2 sequences showed that they clustered in a group provisionally representing a new species [9]. This avastrovirus group also varies because different subgroups can be distinguished $[3,18]$. In recent years, there have been more viruses awaiting formal classification, such as duck hepatitis virus type 2 (DHV-3), DHV-3-like astroviruses, duck astrovirus CPH (DAstV CPH), duck astrovirus SL-like, duck astrovirus YP-like and astroviruses detected in wild birds [5, 10-12, 22].

This report presents the genetic characterization of a novel astrovirus recently detected in Poland. This CAstV strain was associated with increased mortality of embryos and chicks, as well as weakness and white plumage of hatched chicks, a disease described as 'white chicks' condition [16]. The virus was propagated (isolated) on embryonated specific-pathogen-free (SPF) chicken eggs and then used in experimental reproduction of this condition in SPF layer chickens [14].

We used real-time RT-PCR to identify the viral agent according to previous protocols [17]. Six 10-day-old SPF chicken embryos (VALO BioMEDIA, Germany) were inoculated $(0.2 \mathrm{ml} / \mathrm{egg})$ with $10 \%$ suspension of organ sample homogenate in which $\mathrm{CAst} V$ was detected. The inoculated eggs were incubated at $37{ }^{\circ} \mathrm{C}$ and candled daily for 5 days. After cooling, allantoic fluids and altered organs of embryos were harvested. Applied molecular methods revealed a large amount of $\mathrm{CAstV}$ genome $(\mathrm{Ct}$ value of about 15-16) in these samples. The isolated virus was designated as PL/G059/2014. The allantoic fluid was then used in the experimental reproduction of "white chicks' condition and for extraction of RNA for further molecular characterization. We sequenced a nearly fulllength genome of the viral isolate PL/G059/2014 at the commercial service Genomed Sp. z o.o. (Poland). Library preparation, further investigation and "next-generation sequencing" using an Illumina MiSeq System (Illumina Inc., San Diego, USA) were performed. Reads were assembled into contigs and compared to sequences in the GenBank nucleotide and protein databases using BLASTn/ BLASTx. The CLC Genomics Workbench v7.0 was used for all downstream bioinformatic analyses.

The sequence of the nearly full-length genome of the CAstV PL/G059/2014 strain consisted of 7382 nt, excluding the poly(A) tail, and was $301 \mathrm{nt}$ shorter than the full sequence of the reference CAstV GA2011 strain (GenBank accession no. JF414802). The nucleotide composition of the nearly full-length sequence of the CAstV strain is $31 \% \mathrm{~A}, 12 \% \mathrm{G}, 45 \% \mathrm{~T}$ and $12 \% \mathrm{C}$. The G/C content is $24 \%$. NCBI Sequin was used for ORF prediction and genome annotation (Fig. 1).

The PL/G059/2014 had a typical AstV genome structure with three sequential, overlapping ORFs: ORF1a (8-3427, corresponding to positions $22-3441$ of the reference GA2011 sequence), OFR1b (3409-4968, positions 3423-4982 of the reference GA2011 sequence) and ORF2 (4993-7155, positions 5007-7169 of the GA2011 sequence). The nearly complete genome sequence contained a partial $5^{\prime}$ untranslated region (UTR) (nt 1-7, positions 15-21 of the reference GA2011 sequence), and 3'UTR (nt 7156-7382, positions 7315-7521 of the reference GA2011 sequence). Examination of the first nucleotides of the virus revealed the presence of two in-frame AUG codons of ORF1a, beginning at position 8 and 20, respectively (GCGATGGCC CAGGCCATGG8-23), both with the strongest positive effects on translation. ORF1a is $3427 \mathrm{nt}$ long and encodes a polypeptide of 1139 amino acids (aa) with a calculated $\mathrm{M}_{\mathrm{r}}$ of $129.77 \mathrm{kDa}$. As in the case with other astroviruses, there is an overlap region between ORF1a and ORF1b (nt 3409 to 3427), which contains the heptameric frameshift sequence AAAAAAC (nt 3418-3424) known as RFS (ribosomal frameshift signal). ORF1b is $1559 \mathrm{nt}$ long and encodes a polypeptide of 519 aa with a calculated $\mathrm{M}_{\mathrm{r}}$ of $60.41 \mathrm{kDa}$. As is typical for astroviruses, a 24-nt spacer between the stop codon of ORF1b and the start codon of ORF2 with a highly conserved CCGAA pentamer at positions 4980-4984 is also present. ORF2 is $2162 \mathrm{nt}$ long and encodes a capsid protein precursor of 720 aa with a calculated $\mathrm{M}_{\mathrm{r}}$ of $80.01 \mathrm{kDa}$. Rfam analysis of (http://rfam.xfam.org) revealed the presence of a highly conserved coronavirus $3^{\prime}$ stem-loop-II-like motif (s2m) consisting of $23 \mathrm{nt}$ of ORF and the adjacent 18 $n t$ of the $3^{\prime}$ UTR (7136-7178). The exact role of s2m remains obscure, but recently, it was described as genetic element that, through an RNA-interference-like mechanism, influences gene expression in the infected organism, providing some kind of selective advantage for the virus [21].

The predicted nonstructural proteins of PL/G059/2014 contained the characteristic aa motifs that are conserved in other astroviruses: a serine protease (Pro) motif at position 691 (GNSG), a nuclear localization signal (NLS) motif at position 802 (KKKGKTK), and four RdRp motifs at positions 266 (DWTRFD), 328 (GNPSG), 378 (YGDD), 406 (FGMWVK). Six possible transmembrane domains (TM) were detected in the ORF1a protein at the following positions: $220-240$, 375-395, 414-436, 444-462, 477-495, and 660-686.

A phylogenetic analysis of the nearly complete nucleotide sequence was conducted to investigate the relationship of CAstV PL/G059/2014 to other astroviruses. The amino acid sequences of all three ORFs were also compared phylogenetically. All analyses were performed 


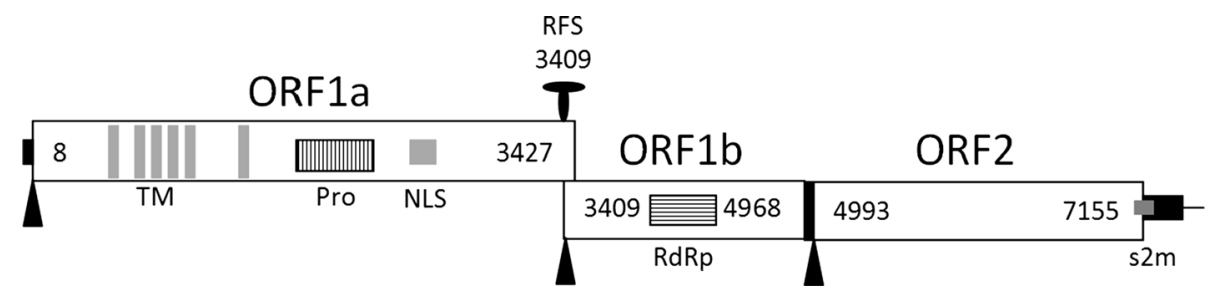

Fig. 1 Predicted genome organization of CAstV PL/G059/2014. Three ORFs with their location and the motifs typical for astroviruses are shown. The translation start sites of ORFs are indicated by black triangles. Black bars are the untranslated regions and the 24-nt spacer between the stop and start codons of ORF1b and ORF2, respectively.

using MEGA version 6.06 [20]. The ClustalW method was used for nucleotide and deduced amino acid sequences alignments, and the neighbour-joining method with 1000 bootstrap replicates was used for generation of phylogenetic trees. Nucleotide and aa sequences of available AstV genomes, representative of putative avian and non-avian astrovirus species, were obtained from GenBank and included in the analysis.

Analysis of the nearly complete nucleotide sequence revealed that PL/G059/2014 was in the same branch of the phylogenetic tree as the only two available CAstVs strains, GA2011 and 4175 (Fig. 2a). The same alignments were obtained in ORF1a and ORF1b phylogenetic trees, with PL/G059/2014 most closely related to the CAstVs GA2011 and 4175 (Fig. 2b and c). With regard to ORF2, previous analysis of available CAstVs showed the existence of two chicken astrovirus groups, namely A and B [3, 18]. The above-mentioned CAstVs GA2011 and 4175, along with other ones from the UK and India, formed group B. However, the PL/G059/2014 strain was found to be in group A with CAstVs/P22-18.8.00 and VF08-36 (Fig. 2d). It is unknown whether the other strains of group $A$ in the analysis of individual ORFs clustered similarly to the Polish strain, since there are only ORF2s available in the public domain. Genome sequence comparison confirmed the results obtained in phylogenetic analysis (Table 1).

The nearly full-length nucleotide genome sequence of PL/G059/2014 has the closest similarity to those of CAstVs GA2011 and 4175, at the level of 71.7-73.7 \%. The next astrovirus strains with similarity to PL/G059/2014 were DAstVs $\mathrm{CPH}$ and C-NGB, with nucleotide sequence identity of $56.7 \%$ and $55.8 \%$, respectively (Table 1). The amino acid sequences of ORF1a and ORF1b of PL/G059/ 2014 shared the highest identities of $86.5 \%$ to $93.8 \%$ with the published sequences of CAstVs GA2011 and 4175 as well (Table 1). However, the aa sequence similarity of ORF2 to these chicken astrovirus strains was rather low, at the level of 32.7-35.2\%. The PL/G059/2014 strain had the highest ORF2 aa sequence identity to P22-18.8.00 and VF08-36 strains, and it ranged between $80.8 \%$ and
ORF, open reading frame; TM, transmembrane domain; Pro, protease; NLS, nuclear localization signal; RdRp, RNA-dependent RNA polymerase; RFS, ribosomal frameshift signal; s2m, stem-loopII-motif

$82.1 \%$. The next astrovirus strains with similarity to PL/ G059/2014 were DAstV-2 strains (representative SL5 in Table 1), with $49.4 \%$ nucleotide sequence identity. In turn, the studied strains showed the lowest similarity, at the level of $20.3 \%$, to astroviruses detected in wild aquatic birds in Cambodia and Hong Kong [5].

The different topology of the PL/G059/2014 strain in the phylogenetic trees of ORF1a/ORF1b and ORF2 as well as nt similarity in different genomic regions to different astrovirus strains suggested that recombination among the astroviruses might have occurred in the field. The ORF1a of PL/G059/2014 was most closely related to that of DAstV-1; and ORF1b, to that of DAstV-3. This situation was also observed in ORF1a and ORF1b of other CAstV strains, GA2011 and 4175. However, in the case of these two CastV strains, similarity of ORF2 to that of DastV-3 was also observed. Surprisingly, the ORF2 of PL/G059/ 2014 was more closely related to a duck astroviruses, DastV-2. This may suggest that the nonstructural and structural protein genes of the PL/G059/2014 came from three different ancestor astroviruses, all hosted by ducks. A recombination event between astroviruses with different hosts of origin as one of the main mechanisms of virus evolution was previously suggested $[11,13,19,23]$. Taking into consideration that many different avastroviruses have recently been detected in ducks and that their genomes showed phylogenetic relationships to poultry astroviruses, it seems probable that ducks may play an important role in the epidemiology of astrovirus, similar to the case with avian influenza virus [1].

The mean amino acid genetic distances (p-dist) based on the analysis of the aa sequence of ORF2 with strains belonging to three official avastrovirus species are as follows: $0.576-0.583$ with the Tk/AK/98 and C-NGB strains of Avastrovirus 3, 0.600 with TAstV-1 of Avastrovirus 1, and 0.725 with G4260 of Avastrovirus 2 (Table 2). On the other hand, the p-dist values with chicken astroviruses GA2011 and 4175 were 0.613-0.644, but only 0.178-0.192 with CAstVs P22-18.8.00 and VF08-36 (Table 2). These p-dist values are even lower 

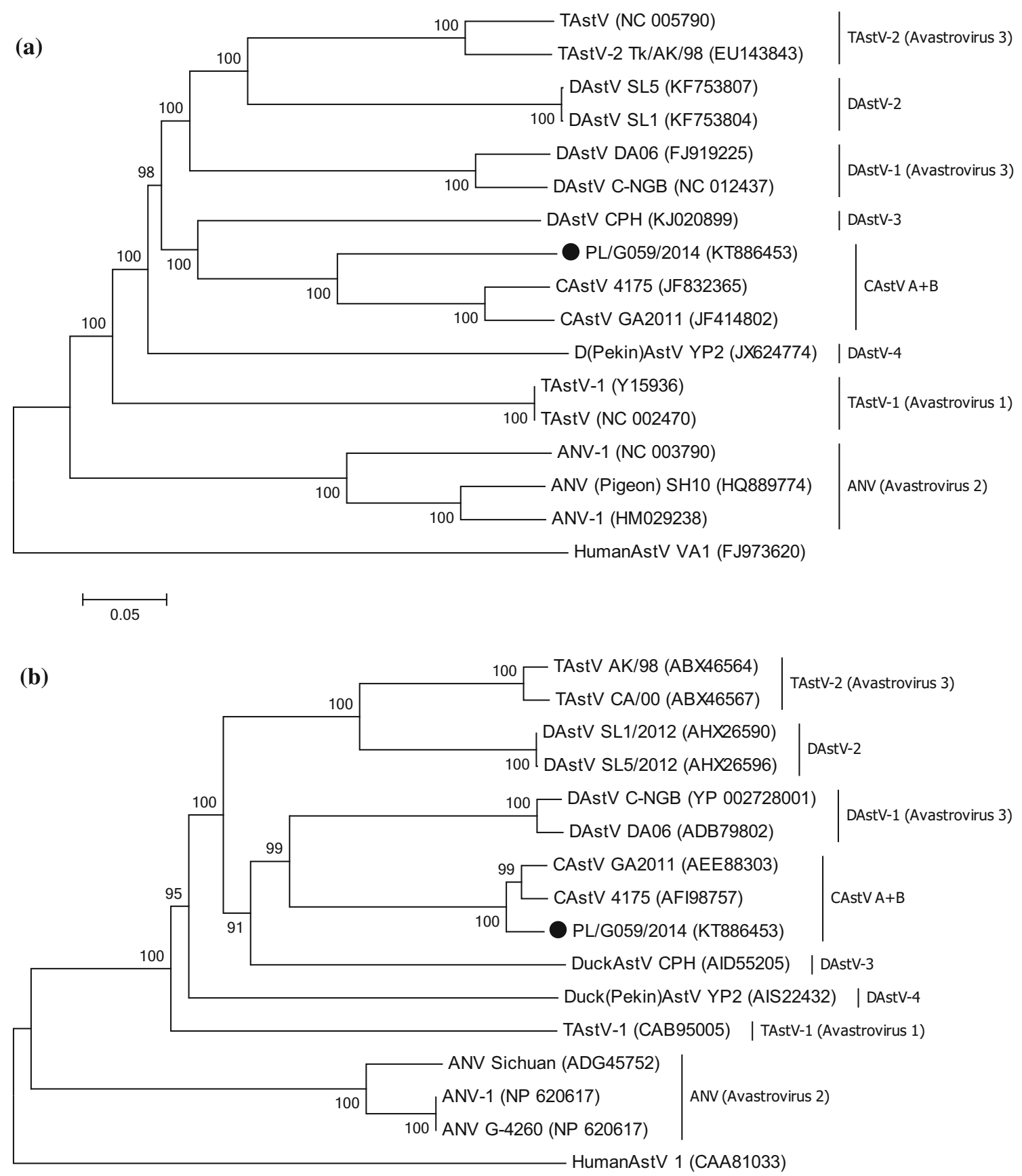

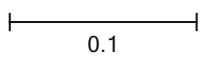

Fig. 2 Phylogenetic relationships between the CAstV PL/G059/2014 isolate from this study and other astroviruses. The analysis was based on the nucleotide sequences of the nearly complete genome (a) and on amino acid sequences of the complete ORF1a (b), ORF1b (c) and ORF2 (d) regions. Human astrovirus (HumanAstV-1) was used as an

than those proposed by the International Committee on Taxonomy of Viruses as criteria for avastrovirus species demarcation (p-dist of 0.576-0.742, and 0.204-0.284 between and within avastrovirus groups), so it seems that strains PL/G059/2014, Dutch P22-18.8.00 and British outgroup. The trees were constructed using MEGA 6, using the neighbor-joining method and 1000 bootstrap replicates (bootstrap values shown on the tree). GenBank accession numbers of the sequences are indicated in parentheses. The PL/G059/2014 isolate determined in this study is indicted by a black dot

VF08-36 belong to the same avastrovirus species but are separate from CAstV GA2011 and 4175 [8].

PL/G059/2014 has been detected in chickens in which the only symptom detected was an impairment in feather pigmentation - the disease known as 'white chicks' 

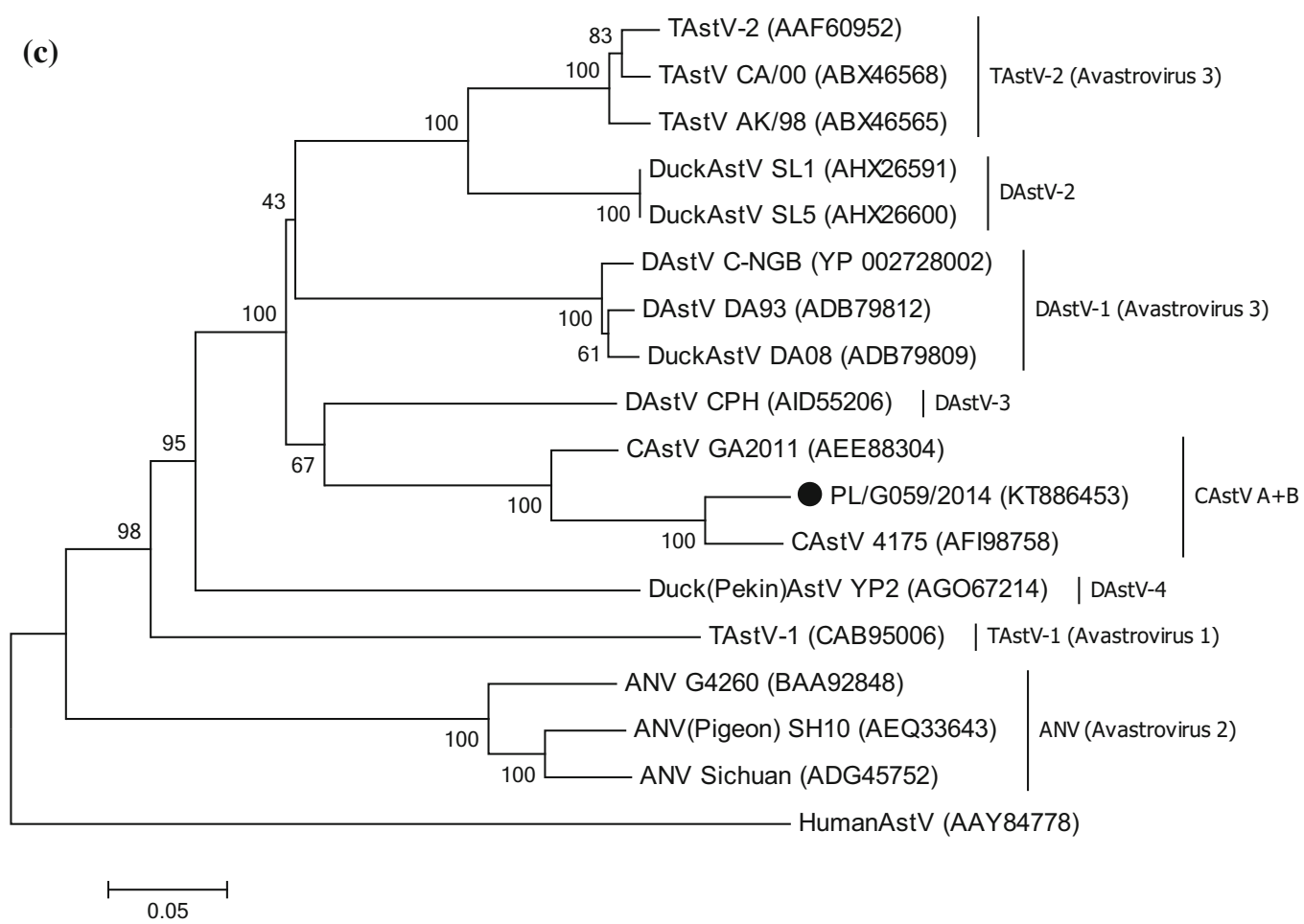

(d)

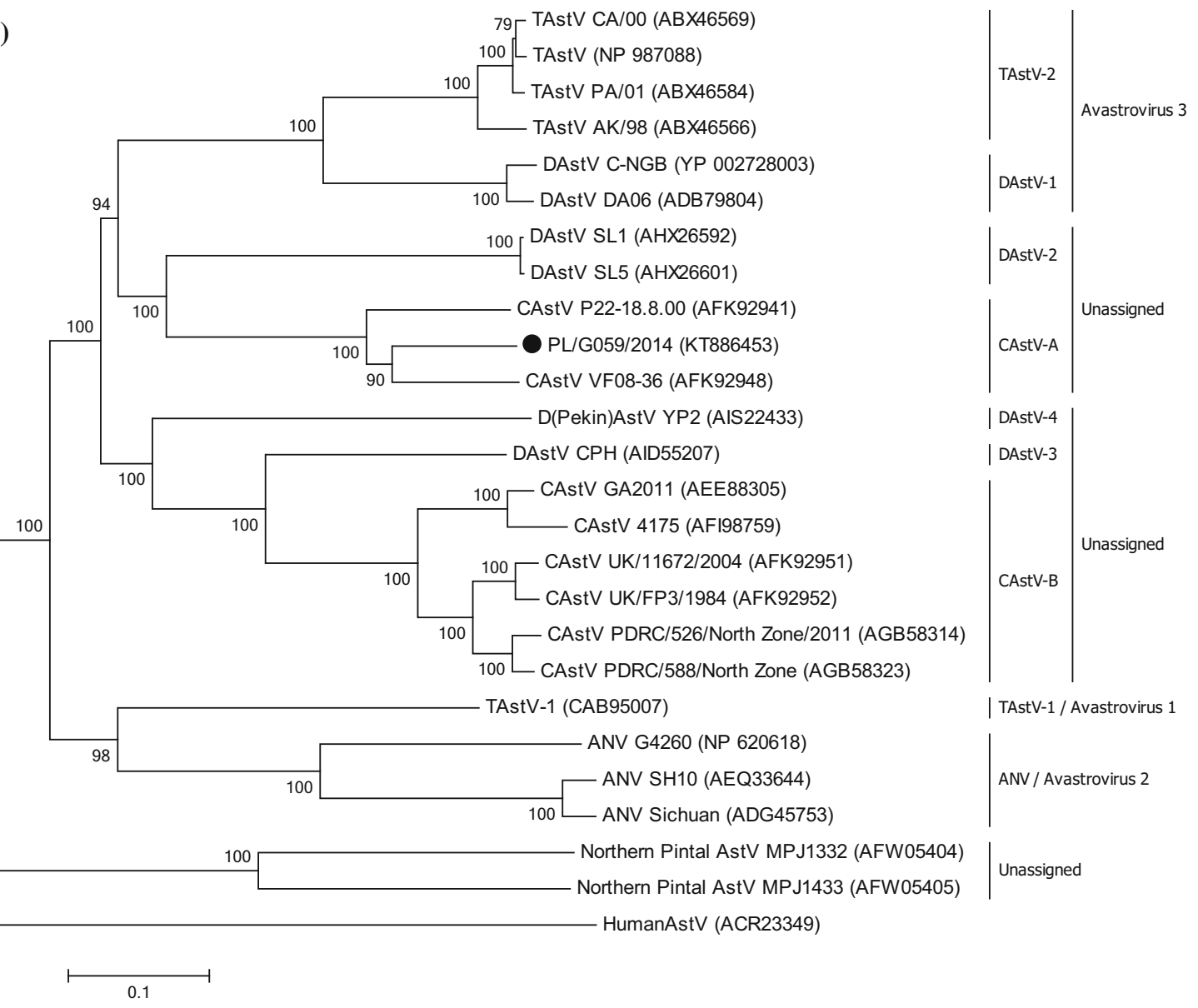

Fig. 2 continued 
Table 1 Evolutionary divergence between sequences

\begin{tabular}{|c|c|c|c|c|c|}
\hline \multirow[t]{2}{*}{ Virus } & \multirow[t]{2}{*}{ GenBank accession number } & \multicolumn{4}{|c|}{ Percent identity to PL/G059/2014 } \\
\hline & & Genome (nt) & ORF1a (aa) & ORF1b (aa) & ORF2 (aa) \\
\hline CAstV 4175 & JF832365 & 73.7 & 93.8 & 93.2 & 32.7 \\
\hline CAstV GA2011 & JF414802 & 71.7 & 93.8 & 86.5 & 35.2 \\
\hline TAstV-2 & EU143843 & 52.8 & 47.4 & 61.8 & 44.3 \\
\hline TAstV-1 & Y15936 & 49.9 & 40.2 & 51.7 & 40.2 \\
\hline ANV-1 & NC003790 & 43.7 & 26.6 & 49.0 & 27.8 \\
\hline DAstV CPH & KJ020899 & 56.7 & 53.3 & 68.1 & 41.9 \\
\hline DAstV SL5 & KF753807 & 53.9 & 48.0 & 65.1 & 49.4 \\
\hline DAstV C-NGB & NC012437 & 55.8 & 58.6 & 62.3 & 43.8 \\
\hline DAstV YP2 & JX624774 & 50.2 & 43.1 & 56.4 & 41.2 \\
\hline BatAstV & EU847155 & 39.8 & 12.8 & 37.1 & 18.1 \\
\hline HumanAstV VA1 & FJ973620 & 37.0 & 13.9 & 34.7 & 19.3 \\
\hline OvineAstV & NC002469 & 36.3 & 15.0 & 34.9 & 18.9 \\
\hline MinkAstV & GU985458 & 36.3 & 15.8 & 34.4 & 18.9 \\
\hline P22-18.8.00 & JN582318 & $\mathrm{n} / \mathrm{a}$ & $\mathrm{n} / \mathrm{a}$ & $\mathrm{n} / \mathrm{a}$ & 80.8 \\
\hline VF08-36 & JN582325 & $\mathrm{n} / \mathrm{a}$ & $\mathrm{n} / \mathrm{a}$ & $\mathrm{n} / \mathrm{a}$ & 82.1 \\
\hline Northern pintal AstV (MPJ1433 isolate) & JX985651 & $\mathrm{n} / \mathrm{a}$ & $\mathrm{n} / \mathrm{a}$ & $\mathrm{n} / \mathrm{a}$ & 20.3 \\
\hline
\end{tabular}

Table 2 Mean amino acid genetic distances (p-dist) based on the analysis of aa sequences of ORF2 of the PL/G059/2014 strain and avian AstV strains of the official species/provisional groups

\begin{tabular}{llr}
\hline Official species/provisional group & Virus strain & p-dist \\
\hline Avastrovirus 3 & Tk/AK/98 & 0.576 \\
Avastrovirus 1 & DAstV C-NGB & 0.583 \\
& TAstV-1 & 0.600 \\
Avastrovirus 2 & G4260 & 0.725 \\
CAstV B & CAstV 4175 & 0.644 \\
& CAstV GA2011 & 0.613 \\
CAstV A & P22-18.8.00 & 0.192 \\
& VF08-36 & 0.178 \\
DAstV 3 & DAstV CPH & 0.581 \\
DAstV 4 & DAstV YP2 & 0.587 \\
DAstV 2 & DAstV SL5 & 0.506 \\
DAstV 1 & DAstV C-NGB & 0.583 \\
Wild birds & Northern Pintal AstV (MPJ1433 isolate) & 0.797 \\
\hline
\end{tabular}

condition. With regard to ORF2 protein structure, the most closely phylogenetically related strains were European CAstV strains that are responsible for enteric and respiratory problems in chickens, as well as hatchability issues $[2,18,22]$. We detected some differences in the capsid protein sequences of these strains, but their implications for pathogenicity are unknown. To investigate this question, whole astrovirus genome sequences should be compared, but they are currently unavailable. However, it should be remembered that pathogenicity could be influenced by the dose and route of inoculation, age and breed of chickens, level of maternally derived or acquired antibodies, and coinfection with other pathogens. For these reasons, conclusions about the pathogenicity of the virus based on their genome sequences should be made with caution.

In conclusion, the present work describes the nearly fulllength genome of chicken astroviruses responsible for 'white chicks' condition recently identified in Poland. Based on the criteria for species demarcation recommended recently by the ICTV, the virus should be classified as a member of a new species within the genus Avastrovirus. Its genomic similarity to different astroviruses supports the previous suggestion that recombination events have played a role in the evolution of astroviruses. The data presented here also suggest a need for redefinition of taxonomic classification criteria for avastroviruses. 


\section{Compliance with ethical standards}

Funding This work was partially funded by KNOW (Leading National Research Centre) Scientific Consortium "Healthy AnimalSafe Food", decision of Ministry of Science and Higher Education No. $05-1 /$ KNOW2/2015.

Conflict of interest The authors declare that they have no conflict of interest.

Ethical approval This article does not contain any studies with human participants or animals performed by any of the authors.

Open Access This article is distributed under the terms of the Creative Commons Attribution 4.0 International License (http://crea tivecommons.org/licenses/by/4.0/), which permits unrestricted use, distribution, and reproduction in any medium, provided you give appropriate credit to the original author(s) and the source, provide a link to the Creative Commons license, and indicate if changes were made.

\section{References}

1. Alexander DJ (2000) A review of avian influenza in different bird species. Vet Microbiol 74:3-13

2. Baxendale W, Mebatsion T (2004) The isolation and characterisation of astroviruses from chickens. Avian Pathol 33:364-370

3. Bulbule NR, Mandakhalikar KD, Kapgate SS, Deshmukh VV, Schat KA, Chawak MM (2013) Role of chicken astrovirus as a causative agent of gout in commercial broilers in India. Avian Pathol 42:464-473

4. Carter MJ, Willcocks MM (1996) The molecular biology of astroviruses. Arch Virol Suppl 12:277-285

5. Chu DKW, Leung CYH, Perera HKK, Ng EM, Gilbert M, Joyner PH, Grioni A, Ades G, Guan Y, Peiris JSM, Poona LLM (2012) A novel group of Avian astroviruses in wild aquatic birds. J Virol 86:13772-13778

6. De Battisti C, Salviato A, Jonassen CM, Toffan A, Capua I, Cattoli G (2012) Genetic characterization of astroviruses detected in guinea fowl (Numida meleagris) reveals a distinct genotype and suggests cross-species transmission between turkey and guinea fowl. Arch Virol 157:1329-1337

7. Gibson CA, Chen J, Monroe SA, Denison MR (1998) Expression and processing of nonstructural proteins of the human astroviruses. Adv Exp Med Biol 440:387-391

8. ICTV Astroviridae Study Group (2011) Reorganize the taxonomy of the genus Avastrovirus (family Astroviridae) into three newly defined species. http://ictvonline.org/proposals/2010.017acV.A.v3. Avastrovirus.pdf. Accessed 28 Feb 2014
9. Kang K, Icard AH, Linnemann E, Sellers HS, Mundt E (2012) Determination of the full length sequence of a chicken astrovirus suggests a different replication mechanism. Virus Genes 44:45-50

10. Liao Q, Liu N, Wang X, Wang F, Zhang D (2015) Genetic characterization of a novel astrovirus in Pekin ducks. Infect Genet Evol 32:60-67

11. Liu N, Wang F, Shi J, Zheng L, Wang X, Zhang D (2014) Molecular characterization of a duck hepatitis virus 3-like astrovirus. Vet Microbiol 170:39-47

12. Liu N, Wang F, Zhang D (2014) Complete sequence of a novel duck astrovirus. Arch Virol 159:2823-2827

13. Pantin-Jackwood MJ, Spackman E, Woolcock PR (2006) Phylogenetic analysis of Turkey astroviruses reveals evidence of recombination. Virus Genes 32:187-192

14. Sajewicz-Krukowska J, Pać K, Lisowska A, Pikuła A, Minta Z, Króliczewska B, Domańska-Blicharz K (2016) Astrovirus-induced "white chicks" condition-field observation, virus detection and preliminary characterization. Avian Pathol 45:2-12

15. Schultz-Cherry SL (2013) Astrovirus infections. In: Swayne DE, Glisson JR, McDougald LR, Nolan LK, Suarez DL, Nair VL (eds) Diseases of poultry, 13th edn. Wiley-Blackwell, Ames, pp 384-396

16. Smyth V, Trudgett J, Wylie M, Jewhurst H, Conway B, Welsh M, Kaukonen E, Perko-Makela P (2013) Chicken astrovirus detected in hatchability problems associated with 'white chicks'. Vet Rec 173:403-404

17. Smyth VJ, Jewhurst HL, Wilkinson DS, Adair BM, Gordon AW, Todd D (2010) Development and evaluation of real-time TaqMan(R) RT-PCR assays for the detection of avian nephritis virus and chicken astrovirus in chickens. Avian Pathol 39:467-474

18. Smyth VJ, Todd D, Trudgett J, Lee A, Welsh MD (2012) Capsid protein sequence diversity of chicken astrovirus. Avian Pathol 41:151-159

19. Strain E, Kelley LA, Schultz-Cherry S, Muse SV, Koci MD (2008) Genomic analysis of closely related astroviruses. J Virol 82:5099-5103

20. Tamura K, Stecher G, Peterson D, Filipski A, Kumar S (2013) MEGA6: Molecular Evolutionary Genetics Analysis version 6.0. Mol Biol Evol 30:2725-2729

21. Tengs T, Kristoffersen AB, Bachvaroff TR, Jonassen CM (2013) A mobile genetic element with unknown function found in distantly related viruses. Virol J 10:132

22. Todd D, Smyth VJ, Ball NW, Donnelly BM, Wylie M, Knowles NJ, Adair BM (2009) Identification of chicken enterovirus-like viruses, duck hepatitis virus type 2 and duck hepatitis virus type 3 as astroviruses. Avian Pathol 38:21-30

23. Todd D, Trudgett J, Smyth VJ, Donnelly B, McBride N, Welsh MD (2011) Capsid protein sequence diversity of avian nephritis virus. Avian Pathol 40:249-259 